\title{
Health Issues in Returned Travellers
}

This chapter will review some of the health issues to consider in returned travellers. While it does not serve as a comprehensive list of all possible causes of illnesses, it focuses on the more common and/or most important illnesses to consider, the types of presentations expected and the place of screening of asymptomatic travellers.

All patients should be asked about whether they have travelled recently. This is always important in the assessment of each patient and has particular relevance in foreign-born patients. A history of travel to developing or tropical countries in the preceding 6 months is especially important for patients presenting with fever or other symptoms suggestive of an infection.

\subsection{Infections in Travellers}

With increasing complexity of travel itineraries, increasing frequency of international travel to tropical regions and increasing numbers of travellers among highrisk groups, such as those with immunosuppression, the elderly, pregnant women and young children, medical practitioners are more often being required to manage more complex travel-related illness. General data are lacking regarding the spectrum of illnesses and the frequency of presentations to general practitioners by Australian returned travellers, but it is estimated that up to one in five travellers seek medical consultation from a general practitioner after returning from abroad. Many ill returned travellers will need to be referred for a specialist opinion, although the urgency of referral varies according to the diagnoses being considered.

The most common infections in returned travellers affect the gastrointestinal and respiratory tracts, followed by cutaneous and sexually transmitted infections. The most common potentially life-threatening travel-related infection is malaria, particularly falciparum malaria, which can progress rapidly unless appropriate treatment is given early; malaria must therefore be excluded in all patients who present unwell after returning from a malaria-endemic area. 
Some less common imported infections may have an importance out of proportion to their incidence because of the need for urgent intervention (e.g. meningococcal infection) or because of their transmissibility and consequent public health implications (e.g. viral haemorrhagic fevers, measles). Consequently, travellersand especially those with a fever-need immediate attention to rule out serious and potentially life-threatening conditions.

Knowledge of the incubation period of particular infections is important. However, many illnesses acquired by travellers will be unrelated to travel or will be due to routine infections acquired during travel rather than an 'exotic' cause. Therefore, assuming all infections occurring after travel abroad are caused by exotic pathogens is not correct and may lead to errors just as serious as those related to ignorance of possible travel-related conditions. Additionally, travellers may return with 'routine' infections (such as urinary tract infections) that are due to multiresistant organisms, and this needs to be considered in the choice of empiric antibiotic therapy.

\subsubsection{Frequency of Illness Among Travellers}

An illness is reported in at least 50\% of travellers to developing countries. The probability of infection is related to a number of factors, including:

- Destination(s)

- Duration of travel

- Reason for travel (e.g. business versus visiting friends and relatives)

- Season

- Type of travel (e.g. backpacking versus five-star accommodation, rural versus urban areas)

- Behaviour

- Specific prophylactic measures such as immunisation and malaria chemoprophylaxis

- Individual susceptibility

It is difficult to determine accurately the actual risks of different infections during travel according to specific itinerary characteristics. However, Table 8.1 shows the kinds of infections seen in returned travellers, and Table 8.2 shows estimates of illness incidence among European and North American travellers to the tropics.

It is also important to remember that the frequency of acquiring certain illnesses during a trip may not correspond to the frequency of presentation with those illnesses following return. For example, data on diarrhoeal illness among studies that evaluate illness occurring during travel show a predominance of bacterial causes of diarrhoea. However, among patients seen after travel, diarrhoea caused by parasites predominates. This is because, if the symptoms persist on return, they are usually more chronic in nature, and chronic diarrhoea is usually caused by different organisms than acute bouts of travellers' diarrhoea that have resolved spontaneously. 
Table 8.1 Reason for seeking medical care among Australian and multinational travellers with febrile illnesses acquired overseas

\begin{tabular}{l|l|l}
\hline & $\begin{array}{l}\text { Percentage of } \\
\text { diagnoses } \\
(n=624)[1]\end{array}$ & $\begin{array}{l}\text { Percentage of } \\
\text { diagnoses } \\
(n=24,920)[2]\end{array}$ \\
\hline Diagnosis & 27 & 21 \\
\hline Malaria & 16 & 14 \\
\hline $\begin{array}{l}\text { Respiratory illness } \\
\text { bacterial pneumonia, } \\
\text { influenza) }\end{array}$ & $\begin{array}{l}(4) \\
(4)\end{array}$ & $(1)$ \\
\hline Gastrointestinal illness & 12 & 15 \\
\hline Dengue fever & 7 & 6 \\
\hline $\begin{array}{l}\text { Typhoid/paratyphoid } \\
\text { (enteric) fever }\end{array}$ & 4 & 2 \\
\hline Rickettsial infection & 3 & 2 \\
\hline Hepatitis A & 1 & $<1$ \\
\hline Fever, no diagnosis & 7 & 22 \\
\hline
\end{tabular}

Table 8.2 Estimates of incidence of health problems per 100,000 European and North American travellers to the tropics per month

\begin{tabular}{l|l}
\hline Any health problem & 45,000 \\
\hline Travellers' diarrhoea (30-80\%) & 35,000 \\
\hline Consulted doctor abroad or back home & 8000 \\
\hline Malaria (no prophylaxis to West Africa) & 2000 \\
\hline Acute febrile respiratory infection & 1400 \\
\hline Influenza A or B & 1000 \\
\hline Giardiasis & 700 \\
\hline Injury & 500 \\
\hline Hepatitis (all types) & 450 \\
\hline Amoebiasis & 400 \\
\hline Tuberculosis & 350 \\
\hline Hepatitis A (300-2000) & 300 \\
\hline Dengue (Southeast Asia) & 200 \\
\hline Animal bite with rabies risk & 200 \\
\hline Gonorrhoea & 200 \\
\hline Malaria (all travellers) & $<100$ \\
\hline Hepatitis B (80-240) & 85 \\
\hline Syphilis & 40 \\
\hline Shigellosis & 20 \\
\hline Typhoid (3-30) & 12 \\
\hline HIV infection & 10 \\
\hline Cholera & 0.3 \\
\hline Meningococcal disease & 0.07 \\
\hline A &
\end{tabular}

Adapted from Steffen et al. [3] and Hill [4]

\subsubsection{History and Physical Examination}

It is essential to take a detailed travel history as this will help to estimate the relative likelihood of potential causative aetiologies. 


\section{Duration of Travel}

For many illnesses that occur in travellers, short at-risk exposure periods (even $<24 \mathrm{~h}$ ) may be sufficient to acquire illness, although increasing travel duration is associated with increased risk of infection. For example, travellers' diarrhoea, malaria, hepatitis A, dengue and enteric (typhoid/paratyphoid) fever can be acquired following journeys with travel durations as short as 1 or 2 days. By contrast, there are some diseases that occur almost exclusively after prolonged or repeated exposures and are therefore rarely seen in short-term travellers. These include filariasis, tuberculosis and leprosy. Many of these infections have long periods of latency and may not present for years after travel.

\section{Precise Travel Itinerary}

Knowing the exact destinations a traveller has visited is critical to compiling an accurate differential diagnosis. Countries vary greatly in disease patterns-for example, yellow fever occurs only in Africa and Latin America, and Japanese encephalitis is confined to parts of Asia. Even within countries there can be focal differences in disease risks according to level of urbanisation, climate, altitude and other factors. For example, malaria in Thailand occurs only in rural areas bordering Cambodia, Laos and Myanmar, but not in Bangkok or in major tourist resorts such as Phuket or Koh Samui. In Indonesia it is common in eastern parts of the country, especially West Irian, but it is rare in Bali. Season of visit is also important in assessing risk.

\section{Type of Exposures}

This includes information about the type of travel undertaken (e.g. backpacking versus business tour) and the quality of the accommodation (e.g. five-star hotel versus hostel/family residence). Knowledge of specific activities undertaken is also important, as this may imply specific risk exposures. For example, the types of food eaten, sexual behaviour, animals encountered, recreational and occupational exposures and contact with insect vectors may be clues to the possible diagnoses (see Tables 5.1-5.4 in Chap. 5).

\section{Vaccination History}

The protective efficacy of immunisations varies considerably and also depends on time since the last dose. A history of up-to-date immunisation with highly efficacious vaccines such as yellow fever, rabies, tetanus, hepatitis A and hepatitis B makes these diagnoses less likely. This is not true for less effective vaccines such as typhoid (approximately $70 \%$ effective) and BCG.

\section{Medications}

Both routine drugs and medications given specifically for the trip may profoundly affect illness. Prophylactic medications may reduce the risk of some infections. In many developing countries, a wide variety of potentially toxic drugs are available over the counter. Travellers often self-medicate with antibiotics, which may modify symptoms and laboratory results without effecting a cure or may themselves cause fever and other symptoms. 


\section{Chronology of Symptoms}

When assessing potential causes of illness, it is crucial to know the duration of travel, the time interval between the patient leaving an endemic area and the onset of illness and the incubation periods of relevant infections. This may allow exclusion of many possibilities without extensive investigations. For example, an incubation period of 3 weeks or more excludes many arboviruses, such as dengue, and viral haemorrhagic fevers. Most travellers present soon after arrival home, with over $90 \%$ of infections related to short-term travel manifesting within 6 months of return. It is rare for illness related to short-term travel to develop more than 1 year later, so a history of recent travel is more likely to be relevant in defining the cause of acute illness than is remote travel. However, history of travel to a malarious area is always relevant.

\section{Medical History}

The risk or severity of illness may be influenced by underlying medical conditions (e.g. malaria is more severe in pregnancy or splenectomised individuals; those on immunosuppressive medication are more likely to develop tuberculosis) or previous exposure (prior hepatitis A provides lifelong immunity).

\section{Physical Examination}

Often, distinctive physical findings will be lacking in returned travellers. However, some findings may increase or decrease the likelihood of certain travel-acquired diseases. For example, the presence of a rash makes the diagnosis of malaria less likely but makes dengue, acute HIV, rickettsial infections and leptospirosis more likely. Hepatomegaly (with or without splenomegaly or jaundice) may be found in conditions such as malaria, viral hepatitis, amoebic liver abscess, enteric fever or brucellosis.

\section{Evaluation of Infections in Returned Travellers}

The approach to assessing the traveller who returns home unwell should make use of signs, symptoms, itinerary details and epidemiology (as outlined above).

If the traveller is unwell, the conditions with the potential for the greatest morbidity and mortality need to be considered and excluded first. For example, if the patient has a fever, the possibility of malaria or septicaemia (e.g. due to typhoid/paratyphoid, meningococcal or pneumococcal sepsis) needs to be assessed immediately.

\subsection{Clinical Syndromes}

\subsubsection{Fever}

The clinical approach to a febrile traveller is in many ways similar to that for any febrile patient. Appropriate steps include assessing the severity and looking for localising symptoms or signs. However, a travel history should lower the threshold for intervention because of the additional possibility of 'exotic' infections. If the traveller has been to a malaria-endemic area, this needs to be considered and excluded promptly with appropriate testing. Blood cultures to diagnose enteric fever and other causes of septicaemia should also be taken immediately. Blood for initial serology for dengue 


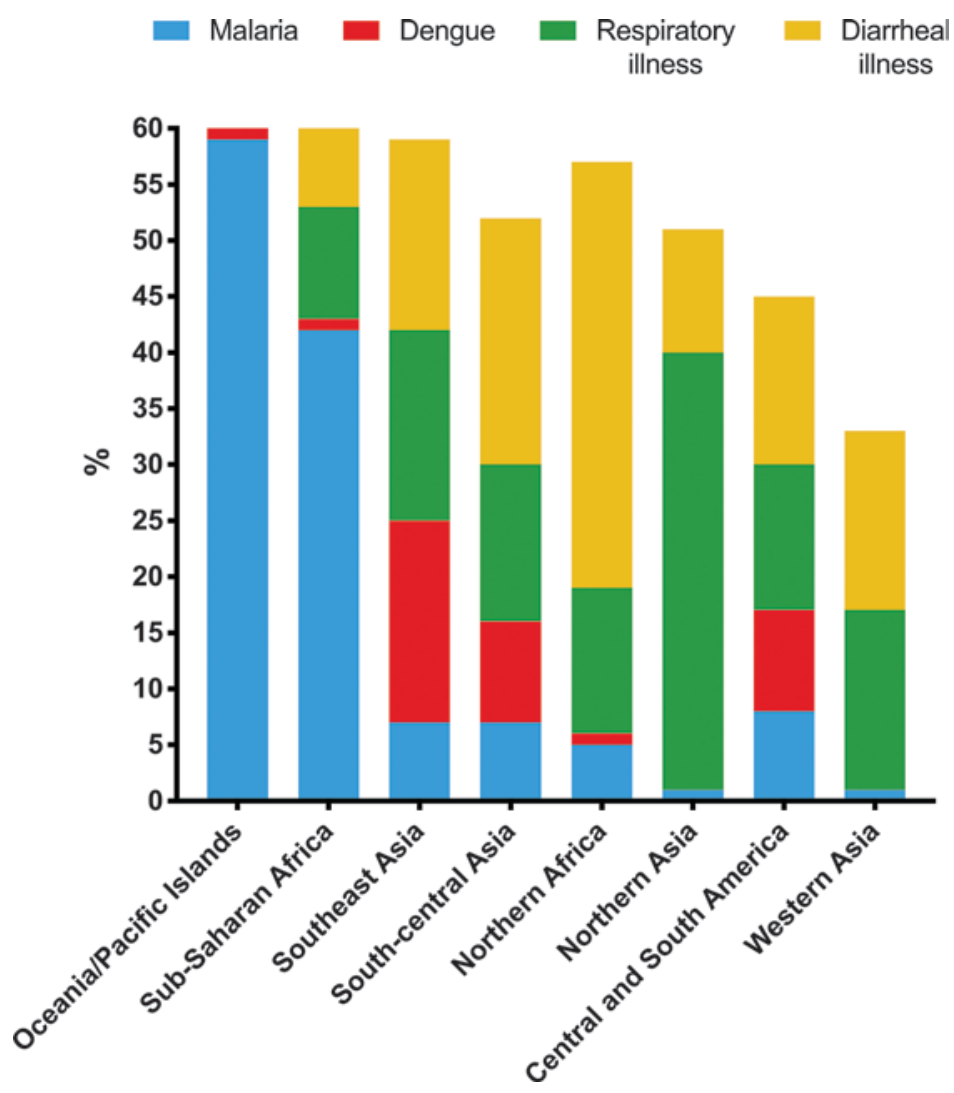

Fig. 8.1 Cause of fever according to region of travel. Relative proportion of febrile travellers from region with diagnosis (adapted from [2])

fever, rickettsial infections and leptospirosis should also be collected at the time of presentation, and again 2-4 weeks later to determine whether or not seroconversion has occurred, confirming recent infection. Most febrile travellers should be hospitalised as this provides the opportunity to monitor the traveller for patterns of fever and the evolution of clinical symptoms and signs and to allow the further collection of relevant blood samples for microbiological microscopy, culture and serological testing.

In attempting to diagnose the cause of the fever, it is important to consider that the most common causes of a febrile illness in returned travellers include malaria, gastroenteritis, upper respiratory tract infections, dengue fever, bacterial pneumonia, influenza, enteric fever, hepatitis A and rickettsial infections (Table 8.1 above, Fig. 8.1). Together, these aetiologies account for over $80 \%$ of the cases of fever in returned travellers.

Fever in a previously healthy young or middle-aged person after travel is most likely to be travel-related. In older individuals, causes unrelated to travel (such as urinary tract infection, pneumonia and intra-abdominal sepsis) become increasingly 
Table 8.3 Incubation periods of imported infections

\begin{tabular}{l|l|l}
\hline Less than 10 days & Intermediate (up to 21 days) & Greater than 21 days \\
\hline Dengue & Malaria & Malaria \\
Influenza & Viral haemorrhagic fever & Viral hepatitis HIV \\
Yellow fever & Rickettsial disease/Q fever & Rabies \\
Chikungunya & African trypanosomiasis & Visceral leishmaniasis \\
Plague & Typhoid fever & Amoebic liver abscess \\
Paratyphoid fevers & Brucellosis & Filariasis \\
Legionella & Leptospirosis & Tuberculosis \\
& Relapsing fever & Q fever \\
& & Acute schistosomiasis \\
\hline
\end{tabular}

Some important diagnoses in febrile returned travellers

likely. These infections may be due to highly resistant pathogens, especially if they occur in the first few weeks after travel. In particular, there is a high chance of having an infection caused by a multiresistant Gram-negative bacteria in travellers returning from Southeast Asia.

In addition to these clinical clues, it is useful to categorise the fever as acute ( $<10$ days), intermediate (10-21 days) or chronic ( $>21$ days) and to assess the likely incubation period (Table 8.3).

\subsubsection{Malaria}

Malaria can mimic other illnesses clinically and may be rapidly fatal if undiagnosed. Essentially any fever in a patient returning from a malaria-endemic area should be considered to be malaria until proven otherwise. The shortest incubation period for malaria is 7-10 days, but presentation can be delayed, particularly in travellers who were taking chemoprophylaxis. For travellers infected with $P$. vivax malaria, it is not unusual for the onset of symptoms to be delayed for more than 1 year. It must be remembered that semi-immune travellers from a malarious area may not be unwell and febrile or may have an additional cause for their fever even if malaria parasites are found in their blood.

\subsubsection{Enteric Fever}

Enteric fever is caused by infection with Salmonella typhi or Salmonella paratyphi. The incubation period is 5-21 days. The common destinations of acquisition include Southeast Asia (particularly Indonesia), South Asia (especially India), Africa, Latin America and the Middle East.

Enteric fever usually presents non-specifically in the early stage with fever, headache, malaise, anorexia, abdominal pain and nonproductive cough. Constipation is more common than diarrhoea in adults. Serious complications include intestinal bleeding/perforation and septic shock. Blood cultures and stool cultures are successful in establishing the diagnosis of enteric fever in the vast majority of 
travellers. Without treatment, the fatality rate is $10-30 \%$, although death in returned travellers is unusual, probably because travellers present to medical practitioners at a relatively early stage of infection. Treatment is usually with a quinolone antibiotic such as ciprofloxacin, but there is increasing resistance to quinolones, especially in isolates from the Indian subcontinent. Alternatives include oral azithromycin or intravenous ceftriaxone.

\subsubsection{Viral Haemorrhagic Fevers (VHF)}

Although uncommon, it is important to consider the possibility of VHF in returned travellers because of the potential public health consequences and the high infectivity of blood, urine and respiratory secretions. The VHFs include Lassa fever, Ebola and Marburg viruses, Congo-Crimean haemorrhagic fever, Rift Valley fever and yellow fever. Clues on history that may suggest their presence include presentation with fever within 3 weeks of travel to a relevant area and exposure, especially if there has been direct contact with blood, body fluids, secretions or excretions of a person or animal with VHF. Clinical findings suggestive of VHF include fever, malaise and myalgia, especially if there are haemorrhagic manifestations in the skin, mucous membranes or internal organs or signs of hypotension and shock (present late in disease). If VHF is suspected, immediate hospitalisation and rapid notification to the communicable disease unit of the local health department are required.

\subsubsection{Dengue Fever, Chikungunya and Zika Virus}

These are discussed in more detail in Chap. 5. These infections have a short incubation period (usually $<5$ days for Chikungunya, 2-7 days for Zika and $<14$ days for dengue fever), and both can cause symptoms including fever, myalgias/ arthralgias and often a rash. The illnesses can sometimes be difficult to distinguish clinically.

\subsubsection{Rickettsial Infections}

These are also discussed in more detail in Chap. 5. Rickettsial infections are comprised of the spotted fever, typhus and scrub typhus groups. Different species occur in different geographic regions and result in illness that is often characterised by fever and rash.

\subsubsection{Diarrhoea}

See Chap. 4.

Diarrhoea is the most common illness that occurs in travellers. The incidence averages 50\% (range: 30-80\%) during the first 2 weeks of travel to developing 
countries. Causes include bacteria, viruses, parasites and toxins. About 10-20\% of diarrhoea involves more than one pathogen, and several discrete episodes of diarrhoea in the same patient may be caused by multiple organisms with different incubation periods acquired during the same contaminated meal.

\section{Acute Diarrhoea ( $\leq 2$ Weeks)}

Most travellers' diarrhoea is brief, self-limited and typically non-inflammatory in type and will have settled by the time of return. The mean duration of symptoms is 4 days; fever and/or bloody diarrhoea occur in 10-20\% of cases. Although a wide range of pathogens are implicated, enterotoxigenic E. coli (ETEC) is the most frequent, being found in $40-70 \%$ of cases when a specific diagnosis is made. The onset of bacterial diarrhoea is usually abrupt. Other commonly identified pathogens include Shigella spp., Salmonella spp., Campylobacter jejuni, Yersinia spp., Vibrio parahaemolyticus, Aeromonas spp., rotavirus and Norwalk-like virus. Remember also that patients with malaria can sometimes present with diarrhoea.

\section{Chronic Diarrhoea (>2 Weeks)}

When diarrhoea persists beyond 14 days, a wider spectrum of causes needs to be considered. Although a bacterial aetiology may still occur, non-ETEC bacteria are likely to be involved. Giardia lamblia is the single most common agent causing chronic diarrhoea. Other causes include Entamoeba histolytica, Cyclospora cayetanensis, Cryptosporidium and Isospora belli. Some helminth infections may also be associated with chronic gastrointestinal symptoms, including hookworm, Ascaris lumbricoides, Trichuris trichiura (whipworm) and Strongyloides stercoralis. Patients with chronic diarrhoea after travel should have their faeces sent for microscopy, culture, parasite examination and PCR testing (for Giardia). Even if no pathogen is demonstrated (including no demonstration of Giardia), all patients with chronic post-travel diarrhoea deserve an empiric trial of therapy for giardiasis. We typically recommend a stat dose of $2 \mathrm{~g}$ tinidazole (Fasigyn) $(50 \mathrm{mg} / \mathrm{kg}$ for children).

\section{Non-infectious Causes}

Travellers' diarrhoea may exacerbate or precipitate symptoms of irritable bowel syndrome or inflammatory bowel disease in the absence of continuing infection. A common diagnosis in returned travellers with persisting gastrointestinal symptoms is 'post-infective irritable bowel syndrome'. No specific therapy is of proven benefit in these cases, although increasing fibre and reducing intake of dairy products and alcohol are often helpful.

\subsubsection{Respiratory Infections}

Respiratory symptoms are reported by up to $30 \%$ of travellers. Respiratory infections are generally considered to be the second most common cause of illness in travellers after travellers' diarrhoea. Some emerging evidence suggests that travel to Asia is associated with an increased risk of acquiring a respiratory infection. 
Many respiratory infections acquired by travellers are caused by common pathogens such as the common cold, influenza or streptococcal pharyngitis. In one study, influenza occurred in almost 3\% of travellers. It should be remembered that, while specific influenza seasons occur in the northern (November-March) and southern hemispheres (April-September), influenza is a year-round disease in the tropics. Seasonal influenza can be imported 'out-of-season' by travellers, and travel is expected to be an important aspect of spread should an influenza pandemic or avian influenza outbreak occur. Consequently, returned travellers who are unwell with suspected influenza should be isolated, and, if admitted to hospital, careful contact and respiratory precautions should be upheld.

It is well accepted that travel played a vital role in the rapid spread of SARS (severe acute respiratory syndrome). Although no community-acquired cases have been reported since 2002, it is possible that new cases could emerge in the future (see Chap. 5). Travel also played an important role in the spread of pandemic influenza A (H1N1) 2009 ('swine flu').

Legionnaires' disease is also common in travellers, particularly to parts of Europe, and may be associated with hotels or spas. Travellers going on cruise ships are also at greater risk of both influenza and legionella infections (see Chap. 5).

Travellers to Saudi Arabia for the Hajj are at extraordinarily high risk of respiratory infections, probably related to the very high level of crowding, which facilitates transmission of respiratory pathogens.

Occasionally respiratory symptoms are due to 'exotic infections', such as acute schistosomiasis (Katayama fever), the migratory phase of Ascaris, strongyloides and/or hookworm infection, acute histoplasmosis, tularaemia and plague. It must also be remembered that both malaria and typhoid can present as fever and cough.

\subsubsection{Skin Problems}

Skin problems occur commonly following travel. The most frequently encountered problems related to travel are sunburn, urticaria, insect bites, swimmer's itch, seabather's eruption, cutaneous larva migrans and myiasis/tungiasis. Additionally, some skin problems such as cellulitis and bacterial abscesses occur more commonly in tropical than temperate regions. Spider bites and envenomations may also occur.

Swimmer's itch is a dermatitis that develops on exposed areas of the skin after contact with waters infested with larval forms of avian schistosomes and if required can be managed symptomatically with topical antipruritic and antihistamine medications. By contrast, seabather's eruption is a dermatitis that appears on covered areas of the skin and is caused by anemone or jellyfish larvae, which typically become trapped underneath bathing suits. Cutaneous larva migrans, also known as creeping eruption, is typically caused by animal hookworm larvae which make contact with human skin during sunbathing (Chap. 5). Myiasis and tungiasis are due to infestation of the skin by fly larvae (see Chap. 5). 
Some systemic infections are also commonly associated with skin changes, such as dengue, rickettsia, acute HIV, leptospirosis, viral haemorrhagic fevers and meningococcaemia. In addition, non-immune travellers may be at increased risk of acquiring measles and rubella when they travel to countries where childhood immunisation rates are lower than in Australia, which include many European countries. If measles is suspected in a returned traveller, public health authorities should be notified rapidly.

\subsubsection{Neurological Infections}

There are a number of important travel-related causes of selected neurological syndromes. Cerebral malaria due to Plasmodium falciparum can cause encephalopathy and coma. Acute meningitis can occur in travellers and may be due to meningococcal infection. Although this is seen rarely in returned travellers, epidemics occur in many countries in Africa, and this infection may also occur in travellers who have visited Saudi Arabia during the Hajj. Acute meningitis may also be due to Lyme disease (caused by Borrelia burgdorferi) and has been seen in returned travellers from North America and Europe. Lyme disease can also be the cause of other neurological syndromes (see Chap. 5).

Chronic meningitis can be caused by tuberculosis or occasionally by brucellosis. HIV and syphilis should also be considered as a cause of meningitis in travellers with a history of unsafe sexual exposures. Eosinophilic meningitis may be caused by certain parasitic infections, including Angiostrongylus cantonensis, Gnathostoma spinigerum and Schistosoma spp. Encephalitis in returned travellers may be due to Japanese encephalitis or other arboviral encephalitides, rabies or African trypanosomiasis (see Chap. 5). Ciguatera and other seafood toxins can also cause neurological presentations.

\subsubsection{Hepatitis}

The most common form of hepatitis acquired by travellers is hepatitis A, which is contracted via exposure to contaminated food or water. It is estimated that unvaccinated travellers to the tropics develop hepatitis A at a rate of 3-20 per 1000 persons per month, but the risk can be almost eliminated by vaccination.

Another virus transmitted via the faecal-oral route that also causes sporadic hepatitis in travellers is hepatitis E. Like hepatitis A, hepatitis E is generally associated with a relatively benign clinical course. However, contracting hepatitis E during the third trimester of pregnancy is associated with up to $20 \%$ mortality of the mother.

The prevalence of hepatitis B is as high as $15 \%$ in some areas, including parts of Southeast Asia, China, Africa, Pacific Islands and the Amazon basin. Overall hepatitis B is three to five times less common than hepatitis A among travellers, but both account for similar mortality. Behaviours involving hepatitis B risk are widespread among travellers. Expatriates, healthcare professionals and individuals 
who have unprotected sexual contacts, share needles, access healthcare services or engage in activities with a high injury risk in endemic areas are at greatest identifiable risk.

Hepatitis $\mathrm{C}$ is a rare infection among returned travellers, but parenteral exposure can occur in areas with a high prevalence of infection, such as Japan, Mediterranean countries, the Middle East and Africa.

A number of other infections can cause hepatitis with or without jaundice, often as part of a systemic illness. These include dengue, yellow fever, malaria, Q fever, amoebic liver abscess and leptospirosis. Some viral causes, particularly the haemorrhagic fever viruses, have limited geographic distribution and, although uncommon in travellers, need to be considered if there is an appropriate exposure history. In addition, drugs that travellers may be taking can be associated with hepatic inflammation.

\subsection{Screening the Returned Traveller Who Is Well}

A question that commonly arises is whether or not well travellers should be screened. It is unnecessary to screen asymptomatic short-term travellers who have not experienced any health problems or have only had trivial self-limited problems such as diarrhoea, as there is no proven benefit of screening these travellers.

For the 'worried well' traveller who has a history of certain high-risk exposures, targeted screening can be considered. For example, individuals who have swum in freshwater lakes known to be infested with schistosomiasis should have serology and urine/faeces for parasite examinations taken (see Chap. 5). For travellers who have had unprotected sex while away, it is appropriate to screen for STIs. Relevant screening tests would include swabs from any site with a discharge, urine PCR for chlamydia and gonorrhoea and serology for hepatitis B, syphilis and HIV. However, knowledge of the prepatent period (time from infection until a laboratory diagnosis can be made) is required in order to be able to rule out the presence of subclinical disease. As examples, the prepatent period for schistosomiasis would be a minimum of 2 months and for HIV about 4-6 weeks.

For longer-term travellers or expatriates, a medical examination is advisable, and it may be appropriate to screen for possible latent infections that may reactivate in the future, such as tuberculosis, strongyloidiasis or chronic asymptomatic infections (e.g. HIV).

\subsection{The Returned Traveller Who Is Unwell/Febrile}

A different approach is required for the traveller who is unwell or febrile on return. The spectrum of infections accounting for the traveller's illness is diverse (Tables 8.1 and 8.2); however, it is important to keep in mind that the most common causes of fever in returned travellers are malaria, gastroenteritis, upper respiratory tract infections, dengue fever, bacterial pneumonia, influenza, enteric fever and rickettsial infections. Nevertheless, several questions must be considered in the assessment 
of an unwell traveller, and these are outlined below. Also, approaching the differential diagnosis by considering fever syndromes (Box 8.1) provides a further useful means of assessing unwell returned travellers.

\section{Box 8.1 Important Diagnoses to Consider in the Unwell Returned Traveller} Fever plus rash

- Dengue fever, Chikungunya, rickettsia, leptospirosis

- Meningococcus, gonococcus, syphilis

- HIV, EBV CMV, measles

- Malaria less likely

\section{Fever plus diarrhoea}

- Travellers' diarrhoea, malaria, typhoid/paratyphoid

\section{Fever plus respiratory symptoms}

- Influenza, common cold, streptococcal pharyngitis/pneumonia, legionnaires' disease, mycoplasma, Q fever

- 'Exotics': tuberculosis, melioidosis and paragonimiasis

- Infiltrates (transient): acute schistosomiasis (Katayama fever), migratory phase of Ascaris, strongyloides or hookworm infection, acute histoplasmosis

\section{Fever plus neurological symptoms}

- Cerebral malaria

- Meningococcal meningitis

- Tuberculous meningitis

- Other exotics: Lyme disease, leptospirosis, brucellosis, melioidosis, cysticercosis, schistosomiasis, Angiostrongylus cantonensis, Gnathostoma spinigerum

- Encephalitis: Japanese encephalitis, other arboviral encephalitis, African trypanosomiasis, rabies, neurosyphilis, HTLV-1 infection

Fever plus jaundice

- Hepatitis A/B/C/E

- Malaria

- Yellow fever, dengue

- Amoebic liver abscess

- Q fever, rickettsia, leptospirosis

\section{Fever and lymphadenopathy}

- Rickettsia

- TB

- Syphilis

- Filariasis

- African trypanosomiasis

- Tularaemia 


\subsubsection{Issues to Consider in the Unwell Returned Traveller}

- Could this be a life-threatening infection? The two diagnoses specifically related to travel that are seen most frequently and need urgent consideration and exclusion are malaria and enteric fever.

- Could it be one of the less common illnesses but potentially fatal if missed and curable if found? These should always be thought of, e.g. meningococcus, legionella, amoebae and melioidosis.

- Is this an uncommon disease but one of public health importance? This includes such illnesses as measles, tuberculosis and viral haemorrhagic fevers.

- Could the fever have subsided but the disease still be present? If not considered, these illnesses will often be overlooked, e.g. acute HIV and schistosomiasis (Katayama fever).

- Could this be an emerging pathogen? As new infections are constantly appearing worldwide, this should always be considered, e.g. SARS.

- Many illnesses acquired by travellers will be unrelated to travel.

- Gastrointestinal and respiratory infections are the most common infections in returned travellers.

- Some imported infections have an importance out of proportion to their incidence because of the need for urgent intervention (e.g. malaria, enteric fever) or because of their transmissibility (e.g. viral haemorrhagic fevers, measles).

- Fever in a returned traveller from malaria-endemic areas must always be assumed to be due to malaria until proven otherwise.

Accurate patient assessment requires a comprehensive travel history encompassing the exact itinerary, mode of travel, patient characteristics, behaviour and immunisations.

\subsubsection{Initial Laboratory Tests to Consider in the Unwell/Febrile Traveller}

- FBE, LFTs, inflammatory markers

- Microscopy and cultures (including examination for ova and parasites): blood, urine, sputum, stool

- Thick and thin smear/ICT test for malaria (three tests should be performed, usually on consecutive days)

- Serology: schistosomiasis, strongyloides, hepatitis, HIV, arboviral, rickettsial, leptospirosis and other

- CXR ( \pm other imaging) 


\section{References}

1. O'Brien D, Leder K, Matchett E, Brown GV, Torresi J. Illness in returned travellers and immigrants: the 6-year experience of 2 Australian infectious diseases units. J Travel Med. 2006;13(3):145-52.

2. Wilson ME, Weld LH, Boggild A, Keystone JS, et al. Fever in returned travelers: results from the GeoSentinel Surveillance Network. Clin Infect Dis. 2007;44:1560-8.

3. Steffen R, Rickenbach M, Wilhelm U, Helminger A, Schär M. Health problems after travel to developing countries. J Infect Dis. 1987;156(1):84-91.

4. Hill DR. Health problems in a large cohort of Americans traveling to developing countries. J Travel Med. 2000;7(5):259-66.

\section{Key Reading}

Angelo KM, Kozarsky PE, Ryan ET, Chen LH, Sotir MJ. What proportion of international travellers acquire a travel-related illness? A review of the literature. J Travel Med. 2017;24(5):tax046.

Artzi O, Sinai M, Solomon M, Schwartz E. Recurrent furunculosis in returning travelers: newly defined entity. J Travel Med. 2015;22(1):21-5.

D'Acremont V, Burnand B, Ambresin A-E, Genton B. Practice guidelines for evaluation of fever in returning travelers. J Travel Med. 2003;10(Suppl 2):S25-52.

Fertitta L, Monsel G, Torresi J, Caumes E. Cutaneous melioidosis: a review of the literature. Int J Dermatol. 2019;58(2):221-7.

Goeijenbier M, van Genderen P, Ward BJ, Wilder-Smith A, Steffen R, Osterhaus AD. Travellers and influenza: risks and prevention. J Travel Med. 2017;24(1). pii: taw078.

House HR, Ehlers JP. Travel-related infections. Emerg Med Clin North Am. 2008;26(2):499-516.

Johnston V, Stockley JM, Dockrell D, Warrell D, et al. Fever in returned travellers presenting in the United Kingdom: recommendations for investigation and initial management. J Infect. 2009;59(1):1-18.

Keystone J, Kozarsky PE, Freedman DO, Nothdurft HD, Commor BA. Travel medicine. 2nd ed. Edinburgh: Mosby; 2007.

Lachish T, Marhoom E, Mumcuoglu KY, Tandlich M, Schwartz E. Myiasis in travelers. J Travel Med. 2015;22(4):232-6.

Morris-Jones R, Morris-Jones S. Travel-associated skin disease. Infect Dis Clin North Am. 2012;26(3):675-89.

O'Brien D, Tobin S, Brown GV, Torresi J. Fever in returned travellers: review of hospital admissions for a 3-year period. Clin Infect Dis. 2001;33:603-9.

Ryan ET, Wilson ME, Kain KC. Illness after international travel. $\mathrm{N}$ Engl $\mathrm{J}$ Med. 2002;347(7):505-16.

Spira AM. Assessment of travellers who return home ill. Lancet. 2003;361(9367):1459.

Steffen R, DuPont HL, Wilder-Smith A. Manual of travel medicine and health. 3rd ed. Hamilton: Decker; 2007.

Thwaites GE, Day NP. Approach to fever in the returning traveler. N Engl J Med. 2017;376(6):548-60.

Ustianowski A, Zumla A. Eosinophilia in the returning traveler. Infect Dis Clin North Am. 2012;26(3):781-9.

Wilson ME, Freedman DO. Etiology of travel-related fever. Curr Opin Infect Dis. 2007;20(5):449-53. 